\title{
Bioenergía a partir de microalgas en México
}

\section{Bioenergy from microalgae in Mexico}

\author{
PÉREZ-BRAVO, Sheila Genoveva †, MENDOZA-MARTÍNEZ, Ana María, CASTAÑEDA-CHÁVEZ, \\ Maria del Refugio y AGUILERA-VÁZQUEZ, Luciano*
} Tecnológico Nacional de México/Instituto Tecnológico de Ciudad Madero, Tamaulipas; Instituto Tecnológico de Boca del
Rio, Veracruz

ID $1^{\mathrm{er}}$ Autor: Sheila Genoveva, Pérez-Bravo / ORC ID: 0000-0001-5333-3763, Researcher ID Thomson: I-5924-2018, CVU CONACYT ID: 728001

ID $1^{\text {er }}$ Coautor: Ana María, Mendoza-Martínez / ORC ID: 0000-0003-2592-9230, Researcher ID Thomson: Q-3436-2017, CVU CONACYT ID: 8374

ID $2^{\text {do }}$ Coautor: Maria del Refugio, Castañeda-Chávez / ORC ID: 0000-0001-8095-6528, Researcher ID Thomson: R-38852017, CVU CONACYT ID: 202403

ID $3^{\text {er }}$ Coautor: Luciano, Aguilera-Vázquez / ORC ID: 0000-0003-2592-9230, Researcher ID Thomson: E-9693-2019, CVU CONACYT ID: 123018

DOI: $10.35429 / J R E .2019 .8 .3 .23 .34$

Recibido 03 de Febrero, 2019; Aceptado 10 Marzo, 2019

\section{Resumen}

La demanda energética de la población mundial va en aumento debido al incremento poblacional y el desarrollo tecnológico. La base energética actual es generadora de emisiones de $\mathrm{CO} 2$, el gas de efecto invernadero más abundante y principal responsable del calentamiento global, así como los gases contaminantes óxidos de azufre y óxidos de nitrógeno. El deterioro ambiental y la alza en los precios de los combustibles fósiles hacen necesaria la investigación de fuentes de energía menos agresivas con el medio ambiente a costos competitivos en el mercado. Los biocombustibles son una alternativa para la producción de energía debido a su origen en el ciclo corto del carbono, sus emisiones se consideran casi nulas, entre ellos se encuentran el biodiésel y bioetanol. Estos últimos pueden ser obtenidos a partir de microalgas ricas en carbohidratos y lípidos, fácil de cultivar en cortos periodos de tiempo. El objetivo de esta investigación es resumir los hallazgos realizados acerca de la existencia de microalgas útiles como materia prima para producir biocombustibles en territorio mexicano. Se realizó una revisión exhaustiva de la bibliografía, que contribuyó estimar la diversidad microalgal en el país y sus contenidos lipídicos así como de carbohidratos, encontrándose distintas especies de los géneros Chlamydomonas, Chlorella, Scenedesmus, Desmodesmus principalmente.

\begin{abstract}
The energy demand of the world population is increasing due to population growth and technological development. The current energy base is a generator of $\mathrm{CO} 2$ emissions, the most abundant and main greenhouse gas responsible for global warming, as well as pollutants, sulfur oxides and nitrogen oxides. The environmental deterioration and the increase in fossil fuel prices make it necessary to investigate less aggressive energy sources with the environment at competitive costs in the market. Biofuels are an alternative for energy production due to their origin in the short carbon cycle, their emissions are considered almost zero, including biodiesel and bioethanol. The latter can be obtained from microalgae rich in carbohydrates and lipids, easy to grow in short periods of time. The objective of this research is to summarize the findings made about the existence of useful microalgae as raw material to produce biofuels in Mexican territory. An exhaustive review of the literature was carried out, which contributed to estimate the microalgal diversity in the country and its lipid contents as well as carbohydrates, with different species of the genera Chlamydomonas, Chlorella, Scenedesmus, Desmodesmus being found mainly.
\end{abstract}

Microalgae, Biofuels, Bioenergy

\section{Microalgas, Biocombustibles, Bioenergía}

Citación: PÉREZ-BRAVO, Sheila Genoveva, MENDOZA-MARTÍNEZ, Ana María, CASTAÑEDA-CHÁVEZ, Maria del Refugio y AGUILERA-VÁZQUEZ, Luciano. Bioenergía a partir de microalgas en México. Revista de Energías Renovables. 2019. 3-8: 23-34

\footnotetext{
*Correspondencia al Autor (Correo Electrónico: luciano.aguilera@itcm.edu.mx)

$\uparrow$ Investigador contribuyendo como primer autor.
} 


\section{Introducción}

En 2016 la población en el país era aproximadamente de 122 millones de habitantes $\mathrm{y}$ por cada uno se requieren $2,126.8 \mathrm{kWh}$ conforme a los indicadores energéticos reportados por la SENER (Secretaría de Energía; Rodríguez et al., 2017; SENER, s/f). Además de la estimación del crecimiento poblacional de personas en el mundo a 9 billones de individuos para el año 2050, se nota un incremento en el consumo de recursos para satisfacer sus necesidades individuales, lo que conlleva una expansión del sector transporte debido a la internalización de la economía y del comercio (Alcalá-Galiano et al., 2018).

El transporte es un sector que aumenta proporcionalmente con el crecimiento de la población, los combustibles requeridos para este sector provienen de la destilación fraccionada de petróleo, compuesto generalmente del $85 \%$ en carbono, $12 \%$ en hidrógeno y $3 \%$ de azufre, nitrógeno y oxígeno.

Debido a su origen fósil es inevitable el arrastre de partículas de azufre, y posteriores emisiones de $\mathrm{SOx}^{1}$, además de $\mathrm{CO}_{2}, \mathrm{NOx}^{2}$, e hidrocarburos no quemados (Medina Ramírez et al. 2012; Concepción-Fandiño y RodríguezMachín, 2018), la utilización de biodiésel en lugar de diésel puede reducir las emisiones de $\mathrm{NOx}^{2}$ y de $\mathrm{SOx}^{1}$, así como contribuir a la reducción de emisiones de gases de efecto invernadero (Tobío-Pérez et al., 2018). La gasolina es el derivado del petróleo con un mayor consumo, ya que es destinado para el funcionamiento de vehículos con motor de encendido provocado y el diésel para motores de encendido a compresión (Rodríguez et al., 2017).

El parque vehicular actual se divide por el tipo de combustible que utilice, siendo los vehículos a gasolina los más utilizados con 97.52 $\%$ y del $2.48 \%$ el total de vehículos diésel en 2015 (SENER, s/f).

Actualmente el mundo enfrenta dos grandes problemas energéticos, la reserva de petrolíferos y la contaminación ambiental causada por la quema de los mismos (FernándezLinares et al., 2012).
La tendencia es la reducción de los combustibles fósiles y reemplazarlos por biocombustibles renovables que no contaminen y resulten en carbono neutro (Medina Jasso et al. 2012; González-Fernández y Ballesteros, 2012; Lin etal., 2018), o por vehículos impulsados con energía eléctrica.

Los biocombustibles con propiedades fisicoquímicas análogas a los derivados del petróleo tienen las ventajas de poder ser utilizados directamente o con algunas modificaciones en el parque automotor actual, como el biodiésel que puede ser almacenado y distribuido en las mismas instalaciones sin requerir cambios importantes en la infraestructura instalada, la tecnología de los motores a compresión no requiere cambios importantes para su utilización. Un estudio realizado por (Tobío-Pérez et al., 2018) evaluó mezclas de diésel-biodiésel y concluye que al usar una mezcla B20 se presenta una disminución de emisiones de $\mathrm{CO}, \mathrm{CO}_{2}$ y $\mathrm{NOx}^{2}$, sin comprometer las prestaciones del motor. Así como el bioetanol puede utilizarse en mezclas con la gasolina como un oxigenante, mejorando la combustión y disminuyendo las emisiones, una mezcla con $10 \%$ de bioetanol en gasolina reduce 3\% las emisiones de Gases de Efecto Invernadero (GEI).

Debido a una combustión más completa se disminuyen las emisiones de $\mathrm{CO}_{2} \mathrm{CO}$ y $\mathrm{NOx}^{2}$, así como de partículas sin quemar mientras se aumenta el octanaje. A este porcentaje de mezcla no se presentan efectos corrosivos en las mangueras, conexiones y tanques de almacenamiento, por otra parte se reporta un incremento en el consumo de combustible del $3.41 \%$ comparado con gasolina de 92 octanos (Andrés, 2018).

El bioetanol producido a partir de caña de azúcar, genera $90 \%$ menos emisiones de gases de efecto invernadero que la gasolina, consumiendo menos del $80 \%$ de energía en la producción (Montiel Montoya, 2010). Los vehículos a gasolina y diésel son una de las principales fuentes móviles de contaminación atmosférica por su contribución de $\mathrm{CO}, \mathrm{NOx}^{2}$ e hidrocarburos (HC) a los inventarios nacionales y locales de emisiones (Llanes Cedeño et al., 2018).

\footnotetext{
${ }^{1}$ Valencia del azufre correspondiente al óxido.
} 
La materia prima principal para la producción de biodiésel son los triglicéridos, utilizar los que provienen de aceites vegetales de cultivos oleaginosas como la soya, girasol y canola se asocia a la afectación de los precios de algunos alimentos (Barajas et al., 2011; Kumar y Sharma, 2016; Ortiz Tapia et al., 2016; Taggar, 2014). Para la producción de bioetanol se utilizan los cultivos amiláceos como la caña de azúcar, maíz, sorgo y remolacha (Alder, 2018; Castro-Martínez et al., 2012), los cuales compiten con el sector alimenticio. Una fuente alternativa de aceites vegetales que no compite con el sector alimenticio son las microalgas.

Las microalgas son organismos celulares microscópicos de 2-200 $\mu \mathrm{m}$, cuyo metabolismo puede ser autótrofo o heterótrofo, incluso mixotrófico. Estos microorganismos se clasifican en: diatomeas, algas verdes, algas verde-azules y algas doradas (Fernández-Linares et al., 2012). Fotoautotróficamente utilizan la luz como fuente de energía y $\mathrm{CO}_{2}$ como fuente de carbono para obtener energía química a través de la fotosíntesis. Heterotróficamente en ausencia de luz utilizan sólo compuestos orgánicos como fuente de energía. Por último mixotróficamente utilizan carbono orgánico e inorgánico como fuente de carbono, la luz y el carbono orgánico son su fuente de energía. Las microalgas almacenan carbohidratos, lípidos y proteínas (Chew et al., 2017; SacramentoRivero et al., 2010), las microalgas verdes generalmente almacenan almidones y carbohidratos, mientras que las cianobacterias acumulan glucógeno. Por otro lado las diatomeas contienen un mayor porcentaje de lípidos, sus porcentajes varían entre especies, acumulando entre el $20 \%$ y $50 \%$ en peso seco de lípidos (Medina Jasso et al., 2012; Alder, 2018). Para su aprovechamiento a nivel laboratorio o industrial es necesario tener una cantidad de biomasa microalgal suficiente y esto depende de las condiciones, ya que son muy diversas de acuerdo a la especie y a la cadena carbonada objetivo.

El comportamiento de las microalgas bajo condiciones de estrés fisiológico como la limitación de nitrógeno y fósforo en crecimiento heterotrófico, así como la exposición a altas intensidades lumínicas en fototrofia son estrategias consideradas eficientes para incrementar el contenido de lípidos en algas verdes, particularmente los constituidos por ácidos grasos saturados y monoinsaturados, ideales para la generación de biodiésel.
Otra de las estrategias utilizadas es cosechar la biomasa en la fase estacionaria de crecimiento, en esta fase los lípidos presentan menos insaturaciones en los ácidos grasos que los conforman (Arias Peñaranda et al., 2012).

Entre las ventajas que presentan los cultivos de microalgas para la extracción de lípidos cabe destacar la naturaleza no polar de los mismos, la eficiencia fotosintética del 3 al $8 \%$ comparada con el $0.5 \%$ de las plantas terrestres y los periodos de cosecha inferiores a 10 días comparados con las cosechas de 1-2 veces al año de las plantas terrestres, por otro lado su demanda estequiométrica de $1.7 \mathrm{~kg}$ $\mathrm{CO}_{2} / \mathrm{kg}$ de biomasa contribuye a la mitigación del efecto invernadero provocado por el $\mathrm{CO}_{2}$, adicionalmente la biomasa residual puede ser utilizada en otros procesos (Arias Peñaranda, Martha Trinidad, Martínez Roldán Alfredo de Jesús, 2012), la productividad por hectárea de ácidos grasos es 30 veces mayor para las microalgas con respecto al maíz y la soya, 10 veces superior comparada con la palma africana (Montero-Sánchez et al., 2012), la productividad por hectárea es su ventaja principal. El rendimiento de la biomasa de microalgas varía de 15 a 25 ton/ha/acre comparado con la soya 0.4 ton/ha/año, colza 0.68 ton/ha/año, palma aceitera 3.62 ton/ha/año y Jatropha 4.14 ton/ha/año (Rashid et al., 2014).

El proceso de conversión de la biomasa microalgal a bioenergía dependerá del producto que quiera obtenerse, en la Figura 1. Se muestra un esquema de los procesos que sufren las fracciones lipídicas y de carbohidratos, específicamente azucares para obtener biocombustibles.

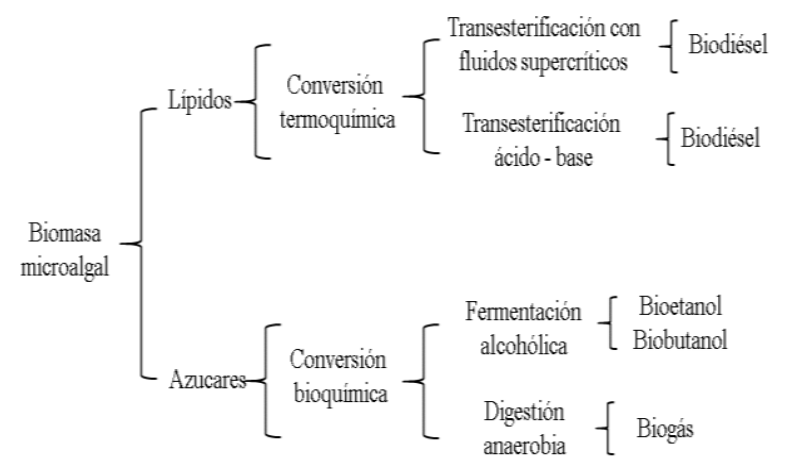

Figura 1 Procesos de generación de biocombustibles Fuente: Basada en (Chew et al., 2017) 
La transesterificación es el proceso químico para la conversión de los triglicéridos haciéndolos reaccionar con un alcohol de cadena corta en presencia de un catalizador ácido, básico o enzimático, obteniendo los ésteres metílicos de ácidos grasos conocidos como biodiésel y el subproducto glicerina. Previo al proceso de transesterificación deben extraerse los lípidos de las microalgas, entre los métodos utilizados se encuentra la extracción con solvente, presión osmótica, extracción isotónica, y enzimática (Chew et al., 2017), algunos investigadores buscan la eliminación de esta etapa realizando transesterificación in situ o directa (Cao et al., 2013; Macías-Sánchez et al., 2015; Sivaramakrishnan y Incharoensakdi, 2018). Generalmente para la producción de biodiésel a partir de microalgas la metodología seguida es la extracción de lípidos, transesterificación y purificación (ArmasMartínez et al., 2019).

El bioetanol a partir de microalgas es obtenido partiendo de un pretratamiento para realizar la sacarificación, entre los que se encuentran los químicos (hidrólisis ácida o alcalina, supercrítica con $\mathrm{CO}_{2}$ ), enzimáticos y mecánicos (homogeneización a alta presión, autoclavado y ultrasonicación). La conversión de sacáridos a bioetanol se puede realizar por los procesos de fermentación oscura, foto fermentación, hidrolisis y fermentación separadas, sacarificación y fermentación simultáneas, así como, sacarificación y cofermentación simultáneas (Kee et al., 2018).

Entre los organismos fermentadores más utilizados se encuentra la levadura Saccharomyse cerevisiae (Marulanda López et al., 2017), Pichia stipitis, Zymomonas mobilis, Kluyveromyces fragilis, Kluyveromyces marxianus, Escherichia coli y Klebsiella oxytoca (Kee et al., 2018). El fermento debe ser destilado a $78^{\circ} \mathrm{C}$, seguido de una deshidratación para así obtener un bioetanol puro y anhidro. Los carbohidratos presentes en las microalgas alcanzan $50 \%$ en peso seco, entre ellos la celulosa y el almidón sin lignina, además de glucosa y polisacáridos es decir son una materia prima para la generación de bioetanol y biobutanol, o bien de biogás. Una de las rutas bioquímicas para la obtención de biocombustibles es la digestión anaerobia, en la cual la biomasa orgánica produce biogás, que contiene metano y $\mathrm{CO}_{2}$ principalmente, el que puede servir directamente como combustible o utilizarse para generar electricidad (Klassen et al., 2016).
Este proceso ha sido utilizado para el tratamiento de residuos orgánicos ya que reduce su carga orgánica además de producir biogás y un sedimento de material orgánico estabilizado con nutrientes mineralizados que puede utilizarse como un fertilizante (Ramos-Suárez y Carreras, 2014).

El proceso de digestión anaerobia se realiza en cuatro fases mediante un complejo conjunto de bacterias y se denominan hidrólisis, acidogénesis, acetogénesis y metanogénesis. Durante estas etapas los biopolímeros complejos se descomponen en oligómeros y monómeros que posteriormente se metabolizan en ácidos grasos de cadena corta y alcoholes, los que se convierten en $\mathrm{CO}_{2}$, hidrógeno y acetato que sirven de sustrato para la fase de metanogénesis que es donde se produce el metano. Las microalgas sirven de materia prima para la digestión anaerobia, se estima que el $50 \%$ de la biomasa de convierte finalmente en biogás (Klassen et al., 2016); entre el 45 y el $85 \%$ de los residuos de extracción de lípidos con solventes de las microalgas también pueden ser digeridos anaerobiamente (Yun et al., 2014).

La biorefinación es el proceso para obtener un conjunto de productos como; biocombustibles, energía y productos de valor agregado (Chew et al., 2017). Una vez obtenido el aceite de las microalgas, los residuos de la biomasa sirven para obtener azucares y bioetanol con rendimientos del $92 \%$ y $93 \%$, respectivamente, o bien pueden ser un sustrato para la producción de biogás o fertilizante (Sivaramakrishnan y Incharoensakdi, 2018).

También se han realizado trabajos, donde la primera etapa consiste en una hidrólisis y fermentación ara obtener bioetanol, y la segunda consiste de una licuefacción de los residuos asistida por bioetanol con rendimientos del $15.84 \%$ en peso de biodiésel, además el sólido residual con $4 \%$ en nitrógeno poder ser utilizado como fertilizante (Rahman et al., 2019). Un proceso rentable de biorrefinación a base de microalgas es necesario para el impulso de los biocombustibles.

Biofields anunció un proyecto de generación de bioetanol a partir de algas verdeazuladas con el apoyo de la Asociación Nacional de Productores de Biocombustibles en 2009 para la adición del 6\% a las gasolinas producidas por Petróleos Mexicanos (PEMEX) (Vilaboa-Arroniz et al., 2019).

PÉREZ-BRAVO, Sheila Genoveva, MENDOZA-MARTÍNEZ, Ana María, CASTAÑEDA-CHÁVEZ, Maria del Refugio y AGUILERAVÁZQUEZ, Luciano. Bioenergía a partir de microalgas en México. Revista de Energías Renovables. 2019 
Sin embargo, hasta el momento la empresa no se cuenta con registro de producción de dicho biocombustible. Biofields es una unidad de negocio del grupo Alejo, dedicada a la genética, agroindustria y Biorrefinación. En Sahuaral, Sonora, se lleva a cabo el cultivo de higuerilla a partir de semillas modificadas para la extracción de aceite y posterior biorrefinación para la obtención de oleoquímicos, reutilizando la pasta de desecho para la producción de forraje y fertilizantes (Giesemann, 2018).

México cuenta con diferentes climas, cuencas, vegetación y topografía, que permiten el desarrollo de una gran diversidad algal, aproximadamente 12 mil cuerpos epicontinentales y ocho lagos naturales (Garduño Solórzano, et al., 2011). Los ríos y arroyos del país constituyen una red hidrográfica de 633 mil km de longitud entre los que destacan 50 ríos principales por los que fluye en $87 \%$ del escurrimiento superficial.

Las cuencas hidrográficas de la república mexicana son 1471 (Semarnat, 2011). La longitud de la línea de costa del océano pacifico es de $7828 \mathrm{~km}$ y del golfo de México y Mar Caribe $3294 \mathrm{~km}$ (INEGI, 2006). En esta investigación se presentan un listado de las microalgas que han sido aisladas en diversas partes del país.

Una reciente evaluación de los recursos naturales de México, las condiciones climáticas, datos geológicos, sitios históricos y urbanos, mediante un Sistema de Información Geográfica (ArcGIS 6.10) y considerando una producción conservadora de microalgas en estanque abierto con capacidad de $20 \mathrm{~g} / \mathrm{m}^{2} \mathrm{~d}$, dan como resultado que el país es capaz de producir 3.8 billones de toneladas de microalgas al año, además considerando las condiciones climáticas, se determinó que el $26.88 \%$ del territorio nacional es adecuado para el cultivo de microalgas, resaltando el potencial de los estados de Jalisco, Oaxaca, Veracruz y Guerrero como los de mayor potencial (Lozano-Garcia, et al., 2019).

Determinar la presencia de las microalgas nativas en una región especifica es un indicativo de la supervivencia de esta en las condiciones ambientales de la misma, en consecuencia, se podrá cultivar el microorganismo en condiciones similares para su posterior conversión a bioenergía.

\section{Metodología}

Se realizó una exhaustiva revisión bibliográfica para resumir cuales son las especies de microalgas halladas en México, así como su contenido de lípidos y carbohidratos, precursores para la producción de biocombustibles en el país, además de los procesos que han sido investigados para la producción de biocombustibles a partir de dichas microalgas.

Estudios han reportado hallazgos de cepas de microalgas en cuerpos de agua del país, tales como la laguna del Carpintero y estero del río Barberena en Tamaulipas, río presidio en Sinaloa, lago de Catemaco en Veracruz y la Bahía Santa Lucia en Guerrero, su presencia indica la capacidad de crecer en dichos ambientes y su adaptación para desarrollarse en las condiciones ambientales.(CrisóstomoVázquez, et al., 2016; Garduño Solórzano, et al., 2011; Hernández-Reyes, et al., 2012; MartínezHernández, et al., 2018; Nájera-Arce, et al., 2018; Ortega-Salas \& Reyes-Bustamante, 2012)

Se reporta el hallazgo de 10 clases de microorganismos y 68 especies, en la Laguna del Carpintero ubicada en Cd. Madero, Tamaulipas (Crisóstomo-Vázquez, et al., 2016), seis de ellas del género Chlorophyceae, presentes a lo largo de los muestreos bimestrales, las microalgas encontradas tienen la característica de almacenar lípidos. Scenedesmus $s p$ tiene la cualidad de almacenar lípidos neutros y polisacáridos de almidón y celulosa sin lignina (Sivaramakrishnan y Incharoensakdi, 2018) por lo cual pueden utilizarse como materia prima para la producción de biodiesel y bioetanol, sin requerir procesos de purificación previos.

En otra reciente investigación se reporta el hallazgo de cuatro especies de microalgas, presentes en agua residual de la zona industrial de Altamira, Tamaulipas, en las que la cepa Chlorella sp. se observa presente en los tres muestreos realizados (Martínez-Hernández, et al., 2018). Por otro lado la especie Chlorella vulgaris fue encontrada en el lago Chalchoapan en la región de los Tuxtlas, Ver. (HernándezReyes, et al., 2012). El género Chlorella se encuentra ampliamente determinado a lo largo del país y sus miembros son capaces de adaptarse a diversas condiciones ambientales y nutricionales en cuerpos de agua dulce y marinos (Moreno et al., 2012; Arias Peñaranda, et al., 2012).

PÉREZ-BRAVO, Sheila Genoveva, MENDOZA-MARTÍNEZ, Ana María, CASTAÑEDA-CHÁVEZ, Maria del Refugio y AGUILERAVÁZQUEZ, Luciano. Bioenergía a partir de microalgas en México. Revista de Energías Renovables. 2019 
Garduño Solórzano y colaboradores (2011) reportan el hallazgo de 17 taxa intraespecíficos, una Cyanoprokaryota y 16 Clorophyta en el Lago de Catemaco, Veracruz, entre las que destacan los géneros Chlorella, Scenedesmus y Desmodesmus, las cuales son ricas en carbohidratos y triglicéridos.

Los cultivos de microalgas pueden realizarse en fotobiorreactores abiertos 0 cerrados, en los que las células de plantas o algas fotoautótrofas se cultivan o se utilizan para llevar a cabo una reacción fotobiológica. Los raceways o lagunas abiertas son sistemas menos caros que los fotobiorreactores cerrados, debido a su menor coste de construcción y operación, teniendo como desventajas una producción de biomasa inferior (Fernández-Linares et al., 2012).

Los géneros de microalgas Chlorella, Scenedesmus, Nannochloris, Dunaliella, Haematococcus, son de interés en la investigación por su contenido de carbohidratos y triglicéridos (Montero-Sánchez et al., 2012; Moreno et al., 2012).

Las especies Chlorella sp., Scenedesmus sp. y S. quadricauda fueron cultivadas en medio CHU10 con y sin nitrógeno, de las cuales se obtuvieron por extracción Soxhlet con una relación cloroformo:metanol $2: 1$, observándose que la tasa de crecimiento fue variable entre las especies cultivadas, y el aumento de la cantidad de lípidos en ausencia de nitrógeno, la producción de biomasa en peso seco fue de $174.7 \mathrm{mg} / \mathrm{l}$ y los lípidos totales de $243.3 \mathrm{mg} / \mathrm{g}$ biomasa seca en medio sin nitrógeno de la especie Scenedesmus sp. Por otro lado Scenedesmus quadricauda y Chlorella sp., mostraron mayor producción de biomasa seca (159.1 mg/g biomasa seca y $221.1 \mathrm{mg} / \mathrm{g}$ biomasa seca respectivamente) en medios con nitrógeno.(Cobos Ruiz, et al., 2016).

Evidentemente a mayor cantidad de triglicéridos mayor producción de biodiésel, el cuello de botella en la transesterificación de lípidos de microalga es la deshidratación de las mismas, este paso genera un gasto energético que encarece el proceso, pudiendo llegar a representar hasta el $30 \%$ de los costos totales (Cao et al., 2013; Macías-Sánchez et al., 2015; Patil et al., 2012).
La disrupción celular de la biomasa microalgal se realiza para acceder con más facilidad a los lípidos intracelulares mediante extracción con solventes, los más utilizados son: hexano, cloroformo, metanol, $\mathrm{CO}_{2}$ supercrítico. Con la finalidad de hacer más eficiente el proceso de transesterificación convencional se han realizado investigaciones más avanzadas, en condiciones supercríticas, con nuevos catalizadores, in situ o con la ayuda de las tecnologías de sonicación y microondas. Queda fuera del alance de este trabajo la descripción detallada y análisis de los procesos de obtención del biodiésel, en la Tabla 3 se mencionan los rendimientos de biodiésel obtenidos a partir de microalgas.

El proceso de fermentación alcohólica requiere de un microorganismo fermentador para convertir los carbohidratos en bioetanol, entre los cuales el más utilizado hasta el momento es Sacaromise cereviase, para el caso de la fermentación de Chlorella sp. se obtiene un $68.59 \%$ de rendimiento para una carga de $100 \mathrm{~g} / \mathrm{L}$, el cual es mejorado al utilizar Pichia stipitis a $74.73 \%$ para la misma carga. Generalmente se ha utilizado la fermentación como segunda etapa en la obtención de bioenergía a partir de microalgas, siendo la primera la extracción de lípidos. Rhaman y colaboradores (2019) proponen realizar la fermentación alcohólica como primer paso y en segundo la licuefacción asistida por etanol para la obtención de biodiésel, obteniendo un rendimiento del $15.84 \%$ para Chlorella sp. afirmando que el rendimiento mejoro un $40.7 \%$ con comparación con la licuefacción de la biomasa original (Rahman et al., 2019). Por otro lado Scenedesmus es conocida por su acumulación de lípidos, carbohidratos y proteínas, del orden del 40, 22.2 y $19 \%$ respectivamente.

Los azucares de esta microalga se pueden extraer con un pretratamiento en autoclave a $121^{\circ} \mathrm{C}$ y 15 psi con $\mathrm{NaOH}$ y $\mathrm{H}_{2} \mathrm{SO}_{4}$ durante 20 min, logrando rendimientos de sacarificación de 82 y $92 \%$. Generalmente los procesos de fermentación alcohólica se realizan después del pretratamiento de hidrolisis ya sea simultáneos o separados alcanzando rendimientos del 68 al $89 \%$ con distintos microorganismos fermentadores, el género de microalga más estudiado es la Chlorella, en la Tabla 4 se observan los rendimientos específicos obtenidos de bioetanol. 
La biomasa microalgal puede ser sometida al tratamiento de digestión anaerobia, en este tipo de experimentos se ha encontrado que la composición de la pared celular de las microalgas tiene un efecto negativo en el rendimiento de metano, ya que algunas presentan resistencia a la degradación en condiciones anaeróbicas. Debido a lo anterior se requiere de un pretratamiento para facilitar la ruptura de las paredes celulares durante la digestión anaerobia (Neves et al., 2016). La aplicación de tratamientos hidrotérmicos son una estrategia efectiva para mejorar la biodegradabilidad de distintas especies de microalgas (Klassen et al., 2016). Al utilizar la biomasa residual de la extracción de lípidos se ha observado que el cloroformo tiene un efecto inhibidor tanto en la producción de hidrógeno como de metano, la biomasa puede ser sometida a un lavado y secado previo a la digestión para disminuir el contenido de cloroformo. En el caso de Chlorella vulgaris se observa un mayor efecto inhibidor en la producción de metano que de hidrógeno cuando la concentración es de 100mg $\mathrm{CHCl}_{3} / \mathrm{L}$ (Yun et al., 2014). Además del cloroformo o sus mezclas cloroformo:etanol para la extracción de lípidos con solventes también se utilizan mezclas de hexano:isopropanol (3:2) y metil pentano, los cuales no presentan efectos inhibidores en la productividad del biometano (Neves et al., 2016; Zhao et al., 2014). Para procesos conjuntos de obtención de energía o bien biorrefinación no es recomendable la extracción de lípidos con cloroformo si el residuo de biomasa será sometido a digestión anaerobia, debido a la inhibición de la metanogénesis.

En un estudio completo elaborado por Klassen y colaboradores (2019) acerca de la digestión anaerobia concluyen que varios factores, entre ellos: las condiciones de cultivo, composición de la biomasa, el momento de la cosecha, pretratamientos, composición química del sustrato, parámetros de digestión anaerobia $(\mathrm{pH}$, temperatura, tasa de carga orgánica, tiempo de retención de sólidos, disponibilidad de nutrientes, potencial de óxido-reducción, presencia de compuestos tóxicos, tamaño de partícula del sustrato y accesibilidad) afectan significativamente la eficiencia del proceso de digestión anaerobia. En este trabajo sólo se mencionan las microalgas que han sido utilizadas con o sin tratamiento para la digestión anaerobia en la Tabla 5, mostrando la productividad de metano por unidad de sólidos volátiles.

\section{Resultados}

En la tabla 1 se muestran las especies de microalgas nativas identificadas en México, así como los cuerpos de agua en los cuales se encontraron.

En la tabla 2 se reportan los contenidos de lípidos y carbohidratos reportados en la literatura para las especies de microalgas más estudiadas hasta el momento

\begin{tabular}{|c|c|c|c|}
\hline Estado & $\begin{array}{l}\text { Cuerpo } \\
\text { de agua }\end{array}$ & Microalga & Referencia \\
\hline \multirow{7}{*}{$\begin{array}{l}\text { Cd. Madero, } \\
\text { Tamps. }\end{array}$} & \multirow{7}{*}{$\begin{array}{l}\text { Laguna } \\
\text { del } \\
\text { Carpintero }\end{array}$} & $\begin{array}{l}\text { Chlamydomonas } \\
\text { reinhardtii }\end{array}$ & \multirow{7}{*}{$\begin{array}{l}\text { Crisóstomo, } \\
\text { et al., } 2016\end{array}$} \\
\hline & & $\begin{array}{l}\text { Chlorella } \\
\text { vulgaris }\end{array}$ & \\
\hline & & $\begin{array}{l}\text { Desmodesmus } \\
\text { denticulatus }\end{array}$ & \\
\hline & & $\begin{array}{l}\text { Desmodesmus } \\
\text { quadricauda }\end{array}$ & \\
\hline & & $\begin{array}{l}\text { Scenedesmus } \\
\text { bijurgus }\end{array}$ & \\
\hline & & Scenedesmus sp. & \\
\hline & & Chaetoceros sp. & \\
\hline \multirow{5}{*}{ Altamira, Tamps. } & \multirow{5}{*}{ A.R.I } & Chlorella sp. & \multirow{5}{*}{$\begin{array}{l}\text { Martínez- } \\
\text { Hernández, } \\
\text { et al., } 2018\end{array}$} \\
\hline & & Lagerheima sp. & \\
\hline & & $\begin{array}{l}\text { Desmodesmus } \\
\text { quadricauda }\end{array}$ & \\
\hline & & Oscillatoria sp. & \\
\hline & & $\begin{array}{l}\text { Scenedesmus } \\
\text { dimorphus }\end{array}$ & \\
\hline Tuxtlas, Ver. & $\begin{array}{l}\text { Lago } \\
\text { Chalchoa- } \\
\text { pan }\end{array}$ & $\begin{array}{l}\text { Chlorella } \\
\text { vulgaris }\end{array}$ & $\begin{array}{l}\text { Hernández- } \\
\text { Reyes, } \\
\text { et al., } 2012\end{array}$ \\
\hline Tampico, Tamps. & $\begin{array}{l}\text { Estero del } \\
\text { Río } \\
\text { Barberena } \\
\end{array}$ & $\begin{array}{l}\text { Spirulina } \\
\text { subsalsa }\end{array}$ & $\begin{array}{l}\text { Hernández- } \\
\text { Reyes, } \\
\text { et al., } 2012\end{array}$ \\
\hline \multirow{3}{*}{ MazatlánSinaloa } & \multirow{3}{*}{$\begin{array}{l}\text { Río } \\
\text { Presidio }\end{array}$} & $\begin{array}{l}\text { Kirchneriella } \\
\text { obesa }\end{array}$ & \multirow{3}{*}{$\begin{array}{l}\text { Ortega- } \\
\text { Salas, et } \\
\text { al., } 2012\end{array}$} \\
\hline & & $\begin{array}{l}\text { Scenedesmus } \\
\text { quadricauda }\end{array}$ & \\
\hline & & $\begin{array}{l}\text { Chlorococcum } \\
\text { infusorium }\end{array}$ & \\
\hline \multirow{11}{*}{ Catema-co, Ver. } & \multirow{11}{*}{$\begin{array}{l}\text { Lago de } \\
\text { Catemaco }\end{array}$} & $\begin{array}{l}\text { Acutodesmus } \\
\text { acuminatus }\end{array}$ & \multirow{11}{*}{$\begin{array}{l}\text { Garduño- } \\
\text { Solorzano, } \\
\text { et al. , } 2011\end{array}$} \\
\hline & & $\begin{array}{l}\text { Closteriopsis } \\
\text { acicularis }\end{array}$ & \\
\hline & & $\begin{array}{l}\text { Oocystis } \\
\text { marssonii }\end{array}$ & \\
\hline & & $\begin{array}{l}\text { Monactinus } \\
\text { simplex }\end{array}$ & \\
\hline & & $\begin{array}{l}\text { Chlorella } \\
\text { minutissima }\end{array}$ & \\
\hline & & $\begin{array}{l}\text { Coelastrum } \\
\text { microporum }\end{array}$ & \\
\hline & & $\begin{array}{l}\text { Pseudanabaena } \\
\text { limnetica, }\end{array}$ & \\
\hline & & $\begin{array}{l}\text { Desmodesmus } \\
\text { quadricauda }\end{array}$ & \\
\hline & & $\begin{array}{l}\text { Scenedesmus } \\
\text { aculeolatus }\end{array}$ & \\
\hline & & $\begin{array}{l}\text { Scenedesmus } \\
\text { obliquus }\end{array}$ & \\
\hline & & $\begin{array}{l}\begin{array}{l}\text { Staurastrum } \\
\text { gracile }\end{array} \\
\end{array}$ & \\
\hline \multirow{3}{*}{ AcapulcoGuerrero } & \multirow{3}{*}{$\begin{array}{l}\text { Bahía } \\
\text { Santa } \\
\text { Lucía del } \\
\text { Puerto de } \\
\text { Acapulco }\end{array}$} & $\begin{array}{l}\text { Chaetoceros } \\
\text { curvisetus, }\end{array}$ & \multirow{3}{*}{$\begin{array}{l}\text { Nájera- } \\
\text { Arce, } \text { et al., } \\
2018\end{array}$} \\
\hline & & $\begin{array}{l}\text { Asterionella } \\
\text { japonica, }\end{array}$ & \\
\hline & & $\begin{array}{l}\text { Biddulphia } \\
\text { mobiliensis }\end{array}$ & \\
\hline
\end{tabular}

Tabla 1 Especies de microalgas en México Fuente: Elaboración Propia

PÉREZ-BRAVO, Sheila Genoveva, MENDOZA-MARTÍNEZ, Ana María, CASTAÑEDA-CHÁVEZ, Maria del Refugio y AGUILERAVÁZQUEZ, Luciano. Bioenergía a partir de microalgas en México. Revista de Energías Renovables. 2019 


\begin{tabular}{|c|c|c|c|}
\hline Microalga & $\begin{array}{l}\text { Lípidos } \\
\text { (\% peso } \\
\text { seco) }\end{array}$ & $\begin{array}{c}\mathrm{HC} \\
(\% \text { peso } \\
\text { seco) }\end{array}$ & Referencia \\
\hline $\begin{array}{l}\text { Chlamydomonas } \\
\text { reinhardtii }\end{array}$ & 21 & 17 & $\begin{array}{l}\text { Spolaore, et } \\
\text { al., } 2006\end{array}$ \\
\hline Chlorella vulgaris & $30-40^{1}$ & $12-17^{2}$ & $\begin{array}{lr}{ }^{1} \text { Liang, et } \\
\text { al.,, 2009 } \\
{ }^{2} \text { Yeh \& } \\
\text { Chang,, } 2012 \\
\end{array}$ \\
\hline Scenedesmus sp. & $\begin{array}{ll}19.6 & - \\
21.1 & \end{array}$ & & $\begin{array}{l}\text { Mata, et } \\
\text { al., } 2010\end{array}$ \\
\hline Chlorella sp. & $28-32$ & & Chisti, 2008 \\
\hline Lagerheima sp. & $42-48$ & & $\begin{array}{l}\text { Martínez- } \\
\text { Hernández } \\
\text { et al., } 2018\end{array}$ \\
\hline $\begin{array}{l}\text { Scenedesmus } \\
\text { dimorphus }\end{array}$ & 31 & & $\begin{array}{l}\text { Shen, et al., } \\
2009\end{array}$ \\
\hline Chlorella vulgaris & $14-22$ & $12-17$ & $\begin{array}{l}\text { Spolaore, } \\
\text { et al., } 2006\end{array}$ \\
\hline $\begin{array}{l}\text { Chlorella } \\
\text { minutissima }\end{array}$ & 31 & 42 & $\begin{array}{l}\text { Illman, et al., } \\
2000\end{array}$ \\
\hline $\begin{array}{l}\text { Scenedesmus } \\
\text { obliquus }\end{array}$ & $12-14$ & $10-17$ & $\begin{array}{l}\text { Spolaore, } \\
\text { et al., } 2006\end{array}$ \\
\hline $\begin{array}{l}\text { Coelastrum } \\
\text { microporum }\end{array}$ & 25 & & $\begin{array}{l}\text { Valdez- } \\
\text { Ojeda, } \\
\text { et al., } 2015\end{array}$ \\
\hline
\end{tabular}

Tabla 2 Contenido de lípidos y carbohidratos Fuente: Elaboración Propia

Cabe mencionar que de los géneros Spirulina y Chaetoceros encontrados en Guerrero y Tamaulipas han presentado características adecuadas para alimentación. El género Spirulina es rico en vitaminas y minerales, entre los que destacan el betacaroteno (provitamina A), vitaminas $\mathrm{E}$ y complejo $\mathrm{B}$, hierro y oligoelementos, dándole un valor comercial, puede producirse biohidrógeno como subproducto (Montiel Montoya, 2010; Colorado, et al., 2013). El género Chaetoceros se ha reportado como alimento de larvas acuaticas (Medina Jasso, et al., 2012).

\begin{tabular}{|l|l|l|l|}
\hline \multicolumn{1}{|c|}{ Microalga } & \multicolumn{1}{|c|}{ Proceso } \\
\hline $\begin{array}{l}\text { Chlorella } \\
\text { pyrenoidosa }\end{array}$ & $\begin{array}{l}\text { Transeste-rificación } \\
\text { in situ }\end{array}$ & $86.8 \%$ & $\begin{array}{l}\text { Cao, et } \\
\text { al., 2013 }\end{array}$ \\
\hline Chrysophyta & $\begin{array}{l}\text { Transeste-rificación } \\
\text { supercríti-ca con } \\
\text { metanol y scCO } \\
\text { como solvente }\end{array}$ & $56.3 \%$ & $\begin{array}{l}\text { Zhou, } \\
\text { et al., } \\
2017\end{array}$ \\
\hline Chlorella sp. & $\begin{array}{l}\text { Transeste-rificación } \\
\text { supercríti-ca con } \\
\text { metanol y scCO } \\
\text { como solvente }\end{array}$ & $63.7 \%$ & $\begin{array}{l}\text { Zhou, et } \\
\text { al., 2017 }\end{array}$ \\
\hline $\begin{array}{l}\text { Nannochloropsis } \\
\text { oculata }\end{array}$ & $\begin{array}{l}\text { Transeste-rificación } \\
\text { heterogé-nea con } \\
\text { Zn0:Mn+2 encapsula- } \\
\text { do en PEG } \\
\text { (Polietilenglicol) }\end{array}$ & $87.5 \%$ & $\begin{array}{l}\text { Vinoth, } \\
\text { et al., } \\
2019\end{array}$ \\
\hline Scenedesmus sp. & $\begin{array}{l}\text { Transeste-rificación } \\
\text { directa catalizada } \\
\text { por enzimas }\end{array}$ & $92 \%$ & $\begin{array}{l}\text { \&ivarama- } \\
\text { \&rishnan } \\
\text { Incharo- } \\
\text { ensakdi, } \\
2018\end{array}$ \\
\hline $\begin{array}{l}\text { Nannochloropsis } \\
\text { gaditana }\end{array}$ & $\begin{array}{l}\text { Transeste-rificación } \\
\text { directa }\end{array}$ & $100 \%$ & $\begin{array}{l}\text { Macías- } \\
\text { Sánchez, } \\
\text { et al., } \\
2015\end{array}$ \\
\hline
\end{tabular}

\begin{tabular}{|l|l|l|l|}
\hline $\begin{array}{l}\text { Nannochloropsis } \\
\text { sp. }\end{array}$ & $\begin{array}{l}\text { Transeste-rificación } \\
\text { extractiva asistida } \\
\text { por microonda }\end{array}$ & $80.1 \%$ & $\begin{array}{l}\text { Patil, } \\
\text { et al., } \\
2012\end{array}$ \\
\hline $\begin{array}{l}\text { Nannochloropsi } \\
\text { s sp. }\end{array}$ & $\begin{array}{l}\text { Transesterificació } \\
\text { n con metanol } \\
\text { supercríti-co }\end{array}$ & $84.1 \%$ & $\begin{array}{l}\text { Patil, et } \\
\text { al., 2012 }\end{array}$ \\
\hline $\begin{array}{l}\text { Chlorella sp. } \\
\text { hidrolizada y } \\
\text { fermentada }\end{array}$ & $\begin{array}{l}\text { Licuefacción } \\
\text { asistida por etanol }\end{array}$ & 15.84 & $\begin{array}{l}\text { Rahman, } \\
\text { et al., } \\
2019\end{array}$ \\
\hline
\end{tabular}

Tabla 3 Producción de biodiésel a partir de microalgas Fuente: Elaboración Propia

\begin{tabular}{|c|c|c|c|}
\hline Microalga & Proceso & Rend. & Referencia \\
\hline $\begin{array}{l}\text { Chlorella } \\
\text { vulgaris }\end{array}$ & $\begin{array}{l}\text { Hidrolisis y } \\
\text { fermentación } \\
\text { separados con } \\
\text { Z. mobilis }\end{array}$ & $87.59 \%$ & $\begin{array}{l}\text { Ho, et al., } \\
2013\end{array}$ \\
\hline $\begin{array}{l}\text { Chlorella } \\
\text { vulgaris }\end{array}$ & $\begin{array}{l}\text { Hidrolisis y } \\
\text { fermentación } \\
\text { simultánea } \\
\text { con } \\
\text { Z. mobilis }\end{array}$ & $87.1 \%$ & $\begin{array}{l}\text { Ho, et al., } \\
2013\end{array}$ \\
\hline $\begin{array}{l}\text { Desmodesmus } \\
\text { sp. }\end{array}$ & $\begin{array}{l}\text { Hidrolisis y } \\
\text { fermentación }\end{array}$ & $81.4 \%$ & $\begin{array}{l}\text { Sanchez } \\
\text { Rizza, et al., } \\
2017\end{array}$ \\
\hline Chlorella sp. & $\begin{array}{l}\text { Hidrolisis } \mathrm{y} \\
\text { fermentación } \\
\text { con } \quad P . \\
\text { stipitis }\end{array}$ & $74.73 \%$ & $\begin{array}{l}\text { Rahman , } \\
\text { et al., } 2019\end{array}$ \\
\hline Chlorella sp. & $\begin{array}{l}\text { Hidrolisis y } \\
\text { fermentación } \\
\text { con } \\
\text { S. cereviase }\end{array}$ & $68.59 \%$ & $\begin{array}{l}\text { Rahman, } \\
\text { et al., } 2019\end{array}$ \\
\hline $\begin{array}{l}\text { Chlorella } \\
\text { vulgaris }\end{array}$ & $\begin{array}{l}\text { Hidrolisis y } \\
\text { fermentación } \\
\text { separados con } \\
\text { S. cereviase }\end{array}$ & $89 \%$ & $\begin{array}{l}\text { Kim, et al., } \\
\text { 2014) }\end{array}$ \\
\hline
\end{tabular}

Tabla 4 Producción de bioetanol a partir de microalgas Fuente: Elaboración Propia

\begin{tabular}{|c|c|c|c|}
\hline Microalga & Pretratamiento & $\begin{array}{l}\text { Produc- } \\
\text { tividad } \\
\text { L CH } \\
\text { kg VS }\end{array}$ & Referencia \\
\hline $\begin{array}{l}\text { Chlorella } \\
\text { vulgaris }\end{array}$ & Ninguno & 337 & \multirow{4}{*}{$\begin{array}{l}\text { Zhao, } \\
\text { et al., } \\
2014\end{array}$} \\
\hline $\begin{array}{l}\text { Chlorella } \\
\text { vulgaris }\end{array}$ & $\begin{array}{l}\text { Lípidos } \\
\text { extraídos }\end{array}$ & 314 & \\
\hline $\begin{array}{l}\text { Nannochloropsis } \\
\text { oculata }\end{array}$ & Ninguno & 357 & \\
\hline $\begin{array}{l}\text { Nannochloropsis } \\
\text { oculata }\end{array}$ & $\begin{array}{l}\text { Lípidos } \\
\text { extraídos }\end{array}$ & 399 & \\
\hline Scenedesmus & Ninguno & 140 & \multirow{3}{*}{$\begin{array}{l}\text { Ramos } \\
\& \\
\text { Carreras, } \\
2014\end{array}$} \\
\hline Scenedesmus & $\begin{array}{l}\text { Aminoácidos } \\
\text { extraídos }\end{array}$ & 272.8 & \\
\hline Scenedesmus & $\begin{array}{l}\text { Lípidos } \\
\text { extraídos }\end{array}$ & 212.3 & \\
\hline
\end{tabular}

Tabla 5 Producción de biogás a partir de la digestión anaerobia de microalgas por unidad de sólidos volátiles. Fuente: Elaboración Propia 


\section{Agradecimiento}

Se agradece a CONACYT por la beca nacional 21542 otorgada al primer autor para cursar el Doctorado en Ciencias de la Ingeniería en el Tecnológico Nacional de México campus Instituto Tecnológico de Ciudad Madero.

\section{Conclusiones}

Entre los generos con mayor porcentaje de lípidos se distingue Chlorella y Lagerheimia, para producir biodiésel se han investigado Chlorella, Scenedesmus y Nannochlorpsis con rendimientos que van desde 56 a $92 \%$ mediante diferentes procesos de conversión.

En cuanto a carbohidratos también se distingue el genero Chlorella, la cual ha sido investigada en la producción de bioetanol así como el genero Desmodesmus con rendimientos de conversión del 68 al $89 \%$.

Además para la producción de biogás pueden ser utilizados los mismos géneros, Chlorella, Scenedesmus y Nannochloropsis, los cuales pueden ser utilizados con o sin tratamiento o bien de forma residual después de haber sufrido la extracción de lípidos o aminoacidos.

Al término de esta investigación se concluye que las microalgas nativas encontradas a lo largo del territorio nacional, especificamente los géneros Chlorella y Scenedesmus halladas en los estados de Sinaloa, Tamaulipas y Veracruz son suceptibles de ser cultivadas y aprovechadas para la producción de los biocombustibles biodiesel, bioetanol y biogás. Además de los géneros Chlamydomonas y Desmodesmus, reportadas como interesantes para la producción de biocombustibles.

Por otro lado se aporta conocimiento de la diversidad microalgal hallada en México, específicamente de las especies Chlamydomonas reinhardtii, Chlorella sp., C. vulgaris, $C$. minutissima, Scenedesmus sp., S. bijurjus, $S$. dimorphus, S. obliquus, S. quadricauda y los géneros Chaetoceros, Biddulphia, Asterionella, Staurastrum, Pseudanabaena, Monactinus, Acutodesmus, Chlorococcum, Kirchneriella, Oscillatoria, de las cuales es necesario continuar investigando su potencial como materia prima para la generación de bioenergía.

\section{Referencias}

Alcalá-Galiano, D., Cujilema-Quitio, M., LeónRevelo, G., Baryolo-González, L., y RamosSánchez, L. (2018). Producción enzimática de biodiesel con biomasa lignocelulósica. Tecnología Química, 38(1), 162-182.

Alder, V. A. (2018). biocombustibles y microalgas marinas. Ciencia e Investigación, 68(1), 29-40.

Andrés, L. Estudio del comportamiento del motor de gasolina chevrolet luv 2200 utilizando bioetanol a partir del residuo de papa con la mezcla E10 (2018). Informe técnico. Universidad Técnica del Norte.

Arias Peñaranda, Martha Trinidad, Martínez Roldán Alfredo de Jesús y Cañizares Villanueva Rosa Oliva (2012). Biodiesel production from microalgae:Cultivation Parameters that Affect Liid Production. N. Biotechnol., 29(1), 43-68.

Armas-Martínez, A. C. D., Morales Zamora, M., Albernas Carvajal, Y., y Erenio, G. S. (2019). Proyección de una industria azucarera para transformarse en una biorrefinería a partir de biocombustibles de segunda y tercera generación. Projection of a sugar industry to become a biorefinery from second and third generation biofuels. Tecnológia Química, 39(3), 489-507.

Barajas, A., González, A., Kafarov, V., Plata, V., Garzón, L., Guzmán, A. y Velásquez, G. (2011). Bioprospección de microalgas colombianas para la producción de biodiesel. Revista Virtual Pro, $1-55$.

Cao, H., Zhang, Z., Wu, X. y Miao, X. (2013). Direct Biodiesel Production from Wet Microalgae Biomass of Chlorella pyrenoidosa through In Situ Transesterification. BioMed Research International, 2013, 1-6.

Castro-Martínez Claudia; Beltrán-Arredondo Laura Ivonne y Ortíz-Ojeda Juan. (2012). Producción de biodiésel y bioetanol: ¿Una alternativa sustentable a la crisis energética? $\mathrm{Ra}$ Ximhai - Revista De Sociedad, Cultura, Desarrollo, 8(3), 93-100.

Chew, K. W., Yap, J. Y., Show, P. L., Suan, N. H., Juan, J. C., Ling, T. C. y Chang, J. S. (2017). Microalgae biorefinery: High value products perspectives. Bioresour. Technol., 229, 53-62.

PÉREZ-BRAVO, Sheila Genoveva, MENDOZA-MARTÍNEZ, Ana María, CASTAÑEDA-CHÁVEZ, Maria del Refugio y AGUILERAVÁZQUEZ, Luciano. Bioenergía a partir de microalgas en México. Revista de Energías Renovables. 2019 
Chisti, Y. (2008). Biodiesel from microalgae beats bioethanol. Trends Biotechnol., 26(3), 126-131.

Cobos Ruiz, M., Paredes Rodríguez, J. D., y Castro Gómez, J. C. (2016). Inducción de la producción de lípidos totales en microalgas sometidas a estrés nutritivo. Acta Biológica Colombiana, 21(1), 17-26.

Colorado, M., Moreno, D., y Pérez, J. (2013). Desarrollo, producción y beneficio ambiental de la producción de microalgas. La experiencia en la Guajira, Colombia. Ambient. y Desarro., 17(32), 113-126.

Concepción-Fandiño, L., y Rodríguez-Machín, L. (2018). Análisis De Lubricidad En Mezcla Diesel-. Revista Iberoamericana de Ingeniería Mecánica, 22(1), 47-53.

Crisóstomo-Vázquez Lilia, Alcocer-Morales Claudia, Lozano-Ramírez Cruz, R.-P. M. C. (2016). Fitoplancton de la Laguna del Carpintero, Tampico, Tamaulipas , México. Interciencia, 41(2), 103-109.

Fernández-Linares Luis Carlos, MontielMontoya Jorge, Millán-Oropeza Aarón, B.-C. A. (2012). Producción de biocombustibles a partir de microalgas. Ra Ximhai - Rev. Soc. Cult. Desarro., 8(3), 543-558.

Garduño Solórzano, G., Rodríguez Palacio, M. C., Martínez García, M., Quintanar Zúñiga, R. E., Lozano Ramírez, C., Campos Contreras, J. E., y Monsalvo Reyes, A. C. (2011). Cultivos de microalgas del Lago de Catemaco , Veracruz. Rev. Latinoam. Biotecnol. Ambient. y Algal, 2(2), 67-80.

Giesemann, R. (2018). Reporte de sustentabilidad 2017-2018. Grupo Alejo.

González-Fernández, C., y Ballesteros, M. (2012). Linking microalgae and cyanobacteria culture conditions and key-enzymes for carbohydrate accumulation. Biotechnology Advances, 30(6), 1655-1661.

Hernández-Reyes, Rodríguez-Palacio, M. C., Lozano-Ramírez, C., y Castilla-Hernández, Y. (2012). Remoción de nutrientes por tres cultivos de microalgas libres e inmovilizados. Rev Latinoam Biotecnol Amb Algal, 3(1), 80-94.
Ho, S., Huang, S., Chen, C., Hasunuma, T. y Kondo, A. (2013). Biore source Technology Bioethanol production using carbohydrate-rich microalgae biomass as feedstock. Bioresour. Technol., 135, 191-198.

Illman, A. . M., Scragg, A. H. H. y Shales, S. W. W. (2000). Increase in Chlorella strains calorific values when grown in low nitrogen medium. Enzyme Microb. Technol., 27, 631-635.

INEGI. Agenda Estadística de los Estados Unidos Mexicanos, Inst. Nac. Estad. Geogr. e Informática. (2006).

Kee, C., Chyuan, H., Chen, W., Chuan, T., y Poh, E. (2018). Overview: Comparison of pretreatment technologies and fermentation processes of bioethanol from microalgae. Energy Conversion and Management, 173, 8194.

Kim, K. H., Choi, I. S., Kim, H. M., Wi, S. G., y Bae, H. J. (2014). Bioethanol production from the nutrient stress-induced microalga Chlorella vulgaris by enzymatic hydrolysis and immobilized yeast fermentation. Bioresource Technology, 153, 47-54.

Klassen, V., Blifernez-Klassen, O., Wobbe, L., Schlüter, A., Kruse, O. y Mussgnug, J. H. (2016). Efficiency and biotechnological aspects of biogas production from microalgal substrates. Journal of Biotechnology, 234, 7-26.

Kumar, M., \& Sharma, M. P. (2016). Selection of potential oils for biodiesel production. Renew. Sustain. Energy Rev., 56, 1129-1138.

Liang, Y., Sarkany, N., \& Cui, Y. (2009). Biomass and lipid productivities of Chlorella vulgaris under autotrophic, heterotrophic and mixotrophic growth conditions. Biotechnol. Lett., 31(7), 1043-1049.

Lin, Y., Ge, J., Ling, H., Zhang, Y., Yan, X. y Ping, W. (2018). Isolation of a novel strain of Monoraphidium sp. and characterization of its potential for A-linolenic acid and biodiesel production. Bioresource Technology, 267, 466472.

Llanes Cedeño, E. A., Rocha-Hoyos, J. C., Peralta Zurita, D. B. y Leguísamo Milla, J. C. (2018). Evaluación de emisiones de gases en un vehículo liviano a gasolina en condiciones de altura. Caso de estudio Quito, Ecuador. Enfoque UTE, 9(2), 149-158.

PÉREZ-BRAVO, Sheila Genoveva, MENDOZA-MARTÍNEZ, Ana María, CASTAÑEDA-CHÁVEZ, Maria del Refugio y AGUILERAVÁZQUEZ, Luciano. Bioenergía a partir de microalgas en México. Revista de Energías Renovables. 2019 
Lozano-Garcia, D. F., Cuellar-Bermudez, S. P., del Rio-Hinojosa, E., Betancourt, F., AlemanNava, G. S. y Parra-Saldivar, R. (2019). Potential land microalgae cultivation in Mexico: From food production to biofuels. Algal Research, 39(February), 101459.

Macías-Sánchez, M. D., Robles-Medina, A., Hita-Peña, E., Jiménez-Callejón, M. J., EstébanCerdán, L., González-Moreno, P. A. y MolinaGrima, E. (2015). Biodiesel production from wet microalgal biomass by direct transesterification. Fuel, 150, 14-20.

Martínez-Hernández Marisol, Suastes-Acosta Sandra L., Lozano-Ramírez Cruz y Rodríguez Palacio. M. C. (2018). Lipidic Profile of Lagerheinia sp. Isolated of Idustrial. Avances en Ciencias e Ingeniería, 9(1), 25-33.

Marulanda López, T., Zapata Osorno, L. F. y Jaramillo Flórez, M. C. (2017). Producción de Bioetanol a partir de Elodea sp. Ingenierías USBmed, 8(1), 37.

Medina Jasso, A., Valdez, P. P., y Soto, M. N. (2012). La importancia de las Microalgas. Biodiversitas, 1-5.

Medina Ramírez E., Chávez Vela A., y Jáuregui Rincón J. (2012). Biodicel, un combustible renovable. Investigación y Ciencia, 55, 62-70.

Montero-Sánchez Yatalí, M.Gómez Liliana, Álvarez Inaudis, C. Sabina Leonor, Támbara Yanet, C. Alfonso Marlén, R. R. L. (2012). Productividad de lípidos y composición de ácidos grasos de cinco especies de miroalgas. Investigación y Saberes, I(2), 37-43.

Montiel Montoya, J. (2010). Potencial y riesgo ambiental de los bioenergéticos en México. Ra Ximhai - Revista De Sociedad, Cultura, Desarrollo, 6, 57-62.

Moreno, M. L. O.-, Castillo, C. E. C.-, SánchezVillarraga, J., Otero-Paternina, A. M., y Padilla, J. (2012). Evaluación del crecimiento de la microalga Chlorella sorokiniana en diferentes medios de cultivo en condiciones autotroficas y mixotroficas. Orinoquia, 16(1), 11-20.

Nájera-Arce, Cynthi; Álvarez-Fitz Patricia; Pérez-Castro, Donaciano; Toribio-Jiménez, Jeiry; Castro-Alarcon, N. (2018). Actividad antibacteriana de diatomeas marinas aisladas de Acapulco, México. Revista de Biología Marina y Oceanografía, 53(2), 195-207.
Neves, V. T. D. C., Sales, E. A., \& Perelo, L. W. (2016). Influence of lipid extraction methods as pre-treatment of microalgal biomass for biogas production. Renewable and Sustainable Energy Reviews, 59, 160-165.

Ortega-Salas, A. A. y Reyes-Bustamante, H. (2012). Cultivo de las microalgas dulceacuícolas Kirchneriella obesa, Scenedesmus quadricauda y Chlorococcum infusorium empleando tres medios de cultivo. Avances en Investigación Agropecuaria, 16(2), 35-44.

Ortiz Tapia, M. del C., García Alamilla, P., Lagunes Gálvez, L. M., Arregoitia Quezada, M. I., García Alamilla, R. y León Chávez, M. A. (2016). Biodiesel production from crude palm oil (Elaeis guineensis Jacq). Ascending path method application. Acta Univ., 26(5), 3-10.

Patil, P. D., Gude, V. G., Mannarswamy, A., Cooke, P., Nirmalakhandan, N., Lammers, P. y Deng, S. (2012). Comparison of direct transesterification of algal biomass under supercritical methanol and microwave irradiation conditions. Fuel, 97, 822-831.

Rahman, Q. M., Zhang, B., Wang, L. y Shahbazi, A. (2019). A combined pretreatment, fermentation and ethanol-assisted liquefaction process for production of biofuel from Chlorella sp. Fuel, 257, 116026.

Ramos-Suárez, J. L. y Carreras, N. (2014). Use of microalgae residues for biogas production. Chemical Engineering Journal, 242, 86-95.

Rashid, N., Ur Rehman, M. S., Sadiq, M., Mahmood, T. y Han, J. I. (2014). Current status, issues and developments in microalgae derived biodiesel production. Renewable and Sustainable Energy Reviews, 40, 760-778.

Rodríguez, L. B., Rionda, R. A., Félix, F. J. C., Ríos, S. B., Miranda, B. H., Hernández, M. P. G., ... Nava, E. V. (2017). Balance nacional de energía 2016. SENER, 136.

Sacramento-Rivero J.C; Romero G.; CortéesRodriguez E.; Blanco-Rosete S. (2010). Diagnostico del desarrollo de biorefinerias en México. A diagnostic study on the development of biorefineries in Mexico. Revista Mexicana de Ingeniería Química, 261-283. 
Sanchez Rizza, Lara; Sanz Smachetti, M. eugenia y D. N. M. (2017). Bioprospecting for native microalgae as an alternative source of sugars for the production of bioethanol. Algal Res., 22, 140-147.

Semarnat. (2011). Atlas del agua en México 2011. Gobierno Federal, 1-142.

SENER. (s/f). SENER | Sistema de Información Energética. Recuperado el 25 de octubre de 2018, de http://sie.energia.gob.mx/

Shen, Y., Pei, Z., Yuan, W. y Mao, E. (2009). Effect of nitrogen and extraction method on algae lipid yield. Int. J. Agric. Biol. Eng., 2(1), 51-57.

Sivaramakrishnan, R. y Incharoensakdi, A. (2018). Utilization of microalgae feedstock for concomitant production of bioethanol and biodiesel. Fuel, 217(January), 458-466.

Spolaore, P., Joannis-cassan, C., Duran, E., Isambert, A., Génie, L. De y Paris, E. C. (2006). Commercial Applications of Microalgae. J. Biosicence Bioeng., 101(2), 87-96.

Taggar, I. S. y M. S. (2014). Recent Trends in Biodiesel Production - An Overview. Applie Engineering Research, 9(10), 1151-1158.

Tobío-Pérez, I., Melo-Espinosa, E. A., SuárezHernández, J., y Ángel, J. (2018). Evaluación de mezclas de biodiésel de Jatropha curcas ( L .) en bancos de motores diésel Evaluation of mixtures of Jatropha curcas ( $\mathrm{L}$.) biodiesel in diesel engine banks Materiales y Métodos. Pastos y Forrajes, 41(4), 300-309.

Vilaboa-Arroniz, J., López-Collado, J., PlatasRosado, D. E., y Vilaboa-Arroniz, I. (2019). El mito de los biocombustibles en México. The myth of biofuels in Mexico. Tropical and Subtropical Agroecosystems, 22, 431-441.

Vinoth Arul Raj, J., Bharathiraja, B., Vijayakumar, B., Arokiyaraj, S., Iyyappan, J. y Praveen Kumar, R. (2019). Biodiesel production from microalgae Nannochloropsis oculata using heterogeneous Poly Ethylene Glycol (PEG) encapsulated $\mathrm{ZnOMn} \mathrm{2+}$ nanocatalyst. Bioresour. Technol., 282, 348-352.
Yeh, K. L. y Chang, J. S. (2012). Effects of cultivation conditions and media composition on cell growth and lipid productivity of indigenous microalga Chlorella vulgaris ESP-31. Bioresour Technol, 105, 120-127.

Yun, Y. M., Cho, S. K., Jung, K. W., Kim, M. S., Shin, H. S. y Kim, D. H. (2014). Inhibitory effect of chloroform on fermentative hydrogen and methane production from lipid-extracted microalgae. International Journal of Hydrogen Energy, 39(33), 19256-19261.

Zhao, B., Ma, J., Zhao, Q., Laurens, L., Jarvis, E., Chen, S. y Frear, C. (2014). Efficient anaerobic digestion of whole microalgae and lipid-extracted microalgae residues for methane energy production. Bioresource Technology, 161, 423-430.

Zhou, D., Qiao, B., Li, G., Xue, S. y Yin, J. (2017). Continuous production of biodiesel from microalgae by extraction coupling with transesterification under supercritical conditions. Bioresource Technology, 238, 609615. 